\title{
IMPROVED POWER-OF-TWO SHARPENING FILTER DESIGN BY GENETIC ALGORITHM
}

\author{
Paolo Gentili, Francesco Piazza and Aurelio Uncini \\ Dip. di Elettronica ed Automatica, University of Ancona \\ Via Brecce Bianche, 60131 Ancona, Italy \\ phone: +39(71) 2204841 fax: +39(71) 2204464 \\ e-mail: aurel@eealab.unian.it
}

\begin{abstract}
For high-speed low-complexity filter design, it is common practice to constrain the filters' coefficients to be power-oftwo or a sum of powers-of-two terms (P2), avoiding the full multiplication.

Tapped interconnection of different sub-filters are sometimes used to enhance ripple and stop-band attenuation performances. An extension of the simple cascade architectures, suitable for hardware implementation, is the polynomial sharpening technique. Firstly proposed by Kaiser-Hamming, it allows the use of identical sub-filters with a small hardware overhead.

In this paper we propose a new approach to the design of P2 sharpening filters based on a specific genetic algorithm which optimizes both the FIR sub-filter and the sharpening polynomial coefficients expressed as P2 terms. This allows to obtain better performances than the classical P2 design techniques when FIR filters with long impulse responses are involved.
\end{abstract}

\section{INTRODUCTION}

In the hardware design of high speed filters, when the coefficients assume power-of-two or a sum of powers-of-two (P2) terms, the filter multiplier can be built using a simple shift register or two shifters plus one adder. So the speed can be very high with a small area occupation. The design of P2 filters, is a very hard combinatorial optimization problem and several sub-optimal algorithms exist for the design of high coarse coefficients FIR filters [4-7].

Differently from the real coefficients case, the basic limitation of the fixed-point or P2 filters, is that, when the filter length is high, increasing the length doesn't affect the performance in terms of pass-band or stop-band ripple. This phenomenon is due to the coefficient quantization error which can be very high especially for small filter coefficients. To overcome this limitation for long filter design, different filter architectures can be used [5], [6].

The filter sharpening techniques [1], [2], [5], in particular, bring to simple hardware architectures, with small number of free parameters, allowing more efficient filter design with better performance especially for long filtes. The basic idea of filter sharpening is the design of an optimized short subfilter to be used as a building-block of more sophisticate polynomial structures. The sub-filter blocks are then combined such that a small hardware overhead is needed [1], [2].

Kaiser and Hamming, in [1] proposed a simple sharpening structure using a suitable polynomial Amplitude Change Function (ACF) that, with some constraint, can be heuristically determined. In [2] Saramaki presented a more general method where the sub-filters and the ACF can be optimized by using a frequency transformation approach. Zhao and Tadokoro, in [5] proposed a method for simple symmetric sharpening (SSS) for P2 filters without polynomial optimization.

In this paper we propose a new P2-filter sharpening technique, based on a specific genetic algorithm (GA) optimized for designing multiplier-less digital filters, which allows the simultaneous optimization of the sub-filter and the sharpening polynomial coefficients, both expressed as power-of-two's.

The new design method, allowing to find global suboptimal solutions for both sub-filters and polynomial structures, can overcome the previous procedure $[1,2,5]$ which instead were able to optimize no more than one circuit at a time. Moreover the proposed procedure is able to find solutions also for very long direct form equivalent filters, letting to design P2 FIR filters with longer impulse response.

Moreover, the use of the GA's, due to their implicit parallel nature, allows to explore many possible solutions at each generation and let the design procedure to be implemented easily and efficiently on parallel machines.

\section{THE DESIGN METHOD}

The sharpening approach consists of combining several identical sub-filters $D(z)$ in a polynomial form $\mathrm{P}[D(z)]$

$$
H(z) \equiv \mathrm{P}[D(z)]=\sum_{i=0}^{M} p_{i} D^{i}(z)
$$


were $M$ is the order of the polynomial, $p_{i}$ are the coefficients, and $H(z)$ is the overall filter response. For infinite precision filter coefficients, the AFC polynomial $\mathrm{P}[D(z)]$ can be built in such a way that it has the $n$ th-order tangency at zero, the $m$ th-order tangency at unity, and passes through the points $(0,0)$ and $(1,1)$. In [1] Kaiser and Hamming proposed the following polynomial $\mathrm{P}[D(z)]$

$$
H(z) \equiv \mathrm{P}[D(z)]=D(z)^{n+1} \sum_{k=0}^{m} \frac{(n+k) !}{n ! k !}(1-D(z))^{k}
$$

Let $\mathbf{p}$ and $\mathbf{d}$ be two arrays containing the power-of-two polynomial and filter coefficients respectively, therefore we assume that the elements of both $\mathbf{p}$ and $\mathbf{d}$ belong to the following domain:

$$
D=\left\{\begin{array}{l}
h: h=\sum_{k=1}^{\lambda} c_{k} 2^{-g_{k}}, \\
c_{k} \in\{-1,0,1\}, g_{k} \in\{-K, \ldots, 0,1, \ldots, B\}
\end{array}\right\}
$$

which is ordered

$$
D=\left\{\alpha_{1}, \alpha_{2}, \ldots, \alpha_{L}\right\} ; \quad\left\{\alpha_{1}<\alpha_{2}<\ldots<\alpha_{L}\right\}
$$

where $\lambda$ is the number of powers of two which forms the coefficients (usually $\lambda=1$ or $\lambda=2$ ), $B$ and $K$ are the integers representing the maximum number of left and right shift that can be performed to avoid the full real multiplication.

Let $d[n], n=0, \ldots,(\mathrm{N}-1) / 2$ be the first half impulse response coefficients of the simmetric sub-filter; the minmax criterion minimizes the maximum error between the filter overall frequency response $H(f)$ and the ideal response $T(f)$ on a dense grid of frequency points $f_{m}, m=1, \ldots, F_{G}$ equally spaced between 0 and 0.5 , i.e. minimizes:

$$
F(\mathbf{x})=\max _{m=1, \ldots, F_{G}}\left\{W\left(f_{m}\right)\left|\left[G^{-1} H\left(f_{m}\right)-T\left(f_{m}\right)\right]\right|\right\}
$$

where $W\left(f_{\mathrm{m}}\right)$ is the weight function, $G \in \mathbf{R}$ is the filter gain factor, $\mathbf{x}$ is an array containing the independent variables such that $\mathbf{x}=[\mathbf{p}|\mathbf{d}| G]$, with $N, F_{G}, \lambda, B$ and $K$ a-priori selected.

For P2 sharpening filters, in order to obtain better performance, we use a filter gain $G$ which can vary in order to minimize the quantization error of both filter and polynomial coefficients, according to the following formula:

$$
F(G)=a_{1} M(G)+a_{2} S_{a}(G)+s_{3} S_{b}(G)
$$

where $G \in[0.5,2.0]$ and $a_{1}=40, a_{2}=3, a_{3}=1$ are determined in order to make the three terms of (5) equal in magnitude. The function $M(G)$ is defined as follows (see [6]):

$$
M(G)=\max _{\mathrm{n} \in[-\mathrm{N}+1, \mathrm{~N}-1]}\left|d[n]-\frac{Q_{D}[G d[n]]}{G}\right|
$$

where $Q_{D}[\bullet]$ represents the operation of rounding to the nearest power-of-two in the domain $D$. The $G$ value which minimizes $M(G)$, allows to reduce the quantization error due to the rounding of the filter coefficients. The functions $S_{a}(G)$ and $S_{b}(G)$ are defined in order to minimize the quantization error due to the rounding of the polynomial coefficients considering that the sharpening polynomial should have a local maximum near the point $(G, 1)$.

The sharpening polynomial is then built similarly to (2) as

$$
H(z) \equiv \mathrm{P}[D(z)]=\left(\frac{D(z)}{G}\right)^{n+1} \sum_{k=0}^{m} \frac{(n+k) !}{n ! k !}\left(1-\frac{D(z)}{G}\right)^{k}
$$

Let $\left(x_{D}, y_{D}\right)$ be the coordinates of the maximum of $\mathrm{P}[D(z)]$ (see Fig. 1), we define the $S_{a}$ and $S_{b}$ as

$$
S_{a}(G)=\left|G-x_{D}\right| ; \quad S_{b}(G)=\left|1-y_{D}\right| \text {. }
$$

A search space reduction can be obtained considering, in accord with (8), the polynomial coefficients as a simple function of the sub-filter $D(z)$.

From the previous assumptions and definitions we can formalise the optimization algorithm as follows:

\section{$\underline{\text { ALGORITHM INITIALISATION }}$}

1. For a given sub-filter of order $N$, determine the infinite precision coefficients, denoted as d', by the Parks-McClellan [3] algorithm and then quantize them as $\mathbf{d}_{\mathbf{0}}=Q_{D^{[}}\left[\mathbf{d}^{\prime}\right]$.

2. Determine the real value $G_{0}$ by the minimizion of (5).

3. Using the expression (8), determine the polynomial coefficients $\mathbf{p}$ using $\left[G_{0} D(z)\right]$ as argument.

The polynomial coefficients are computed by (8) in a deterministic way, so the variable vector $\mathbf{x}$ of the objective function $F(\mathbf{x})$ is simply re-defined as $\mathbf{x}=[\mathbf{d} \mid G]$. The array $\mathbf{x}_{\mathbf{0}}=\left[\mathbf{d}_{\mathbf{0}} \mid G_{0}\right]$ represents the leading value for the optimization algorithm.

\section{FREE PARAMETERS ENCODING}

In the classical GA algorithm the free parameters are directly encoded using a binary (or Gray) string [8], [9], [10]. In the proposed algorithm, in order to reduce the computational cost and to improve the performance, instead of coding directly the $\mathbf{x}$ variable we code a suitable interval, around the leading value $\mathbf{x}_{\mathbf{0}}$, defined as an integer vector $\mathbf{i}_{\mathbf{x}}=\mathrm{I}\left(\mathbf{x}_{\mathbf{0}}\right)$. The 
$\mathbf{i}_{\mathbf{x}}$ contains the indexes of the allowed values for the filter coefficients $\mathbf{d}$ in the domain $D$, and the index of a lessquantized interval around the $G_{0}$ value.

Using such integer codes as genetic strings, a population of quantized filters is created. The evolution then takes place with given probabilities of mutation and crossing-over. The fitness of each individual is computed by expr. (4) and then normalized so that the lowest fitness in the population is set to 0 and the highest to 10000 . To avoid the genetic drift in small populations, we have chosen to use a multiple crossing-over [8] on the genetic strings of two individuals. An elitist mechanism, similar to the De Jong's model [8], has been also implemented, increasing the fitness of the best individuals in the population by a fixed amount roughly proportional to the fitness itself.

After a given number of generations, the performance has been measured by the maximum weighted error $e d B$ expressed in decibel $[5,6]$ :

$e_{d B}=\left[\frac{\delta}{\left(H_{\max }(f)+H_{\min }(f)\right) / 2}\right]_{d B} ;$

where $\delta$ is the peak weighted ripple, $H_{\max }(f)$ and $H_{\min }(f)$ are respectively the maximum and minimum value of the frequency response in the passband.

\section{EXPERIMENTAL RESULTS}

In order to test the proposed method, many linear-phase sharpening FIR filter design experiments have been carried out varying the filter lengths and the initial conditions for the GA. In this paper we report only 3 significant designs already proposed in [5].

Table 1 reports the maximum weighted error in decibel $e_{d B}$ versus the number of taps of the filter. In column two we report the infinite precision filter performance computed by the Remez exchange algorithm [3]. The column three contains the results of the filter design with GA without sharpening. Column five contains the results of infinite precision SSS design. The columns six and seven contain the results of the proposed P2 sharpening design (P2-SSS) and the optimized P2-polynomial sharpening coefficients. All ripples are expressed in $[\mathrm{dB}]$ following the notation (10).

From this results we can observe that for short P2-FIR filter the direct form performs better than the SSS form. As above remarked, on the contrary when the filter length becomes high (greater than 99 taps) the P2-SSS design overcomes the P2-FIR direct form. We can also note that P2-SSS filters perfom better than the infinite precision SSS.

\section{CONCLUSIONS}

A new approach to design P2 sharpening filters has been proposed. It is based on the use of a specific genetic algorithm that, with a particular free parameters encoding around a set of suitable leading values, allows to obtain a very high reduction of the computational cost.

The experimental results show that optimising both the polynomial and the filters coefficients allows to obtain very good performances, sometime better that the simple infinite precision sharpening techniques.

\section{REFERENCES}

[1] J.F. Kaiser, R.W. Hamming, "Sharpening the Response of a Symmetric Nonrecursive Filter by Multiple Use of the Same Filter", IEEE Trans. Acoust. Speech, Signal Processing, Vol. ASSP-25, No.5, pp. 415-422, Oct. 1977.

[2] T. Saramaki, "Design of FIR Filters as a Tapped Cascaded Interconnection of Identical Subfilters", IEEE Trans. on Circuits and Systems, Vol. CAS-34, No.9, pp. 1011-1029, Sept. 1987.

[3] T. W. Parks, C. S. Burrus, "Digital Filter Design", New York, Wiley, 1989.

[4] Y. C. Lim, S. R. Parker, "FIR Filter Design Over a Discrete Powers-Of-Two Coefficient Space", IEEE Trans. Acoust. Speech, Signal Proc., Vol. ASSP-31, pp. 583-591, June 1983.

[5] Q. F. Zhao, Y. Tadokoro, "A Simple Design of FIR Filters with Power-Of-Two Coefficients", IEEE Trans. on Circuits and Systems, Vol. CAS-35, No.5, pp. 566-570, May 1988.

[6] N. Benvenuto, M. Marchesi, A. Uncini, "Applications of Simulated Annealing for the Design of Special Digital Filters", IEEE Trans. on Signal Processing, Vol. 40, pp. 323-332, Feb. 1992.

[7] H. Samueli, "An Improved Search Algorithm for the Design of Multiplierless FIR Filters with Power-ofTwo Coefficients", IEEE Trans. on Circuits and Systems, Vol. CAS-36, pp. 1044-1047, July 1989.

[8] D. E. Goldberg, "Genetic Algorithms in Search", Optimization and Machine Learning, Addison-Wesley, Reading, Mass., 1989.

[9] G. D. Cain, A. Yardim, "Darwinian Design of FIR Digital Filters", Workshop on Genetic Algorithms, Neural Networks and Simulated Annealing, Glasgow, UK, May 1990.

[10] J.D. Schaffer, L.J. Eshelman, "Designing Multiplierless Digital Filters Using Genetic Algorithms", Proc. of Int. Conf. on Genetic Algorithms (ICGA93), pp. 439-443, 1993.

[11] P. Gentili, F. Piazza, A. Uncini, "Efficient Genetic Algorithm Design for Power-of-Two FIR Filters", Proc. of Int. Conf. on Acoustic Speech and Signal Processing (ICASSP95), Detroit-USA, pp.1268-1271, May 1995.

This work is supported by the Ministero dell'Università e della Ricerca Scientifica e Tecnologica of Italy. 

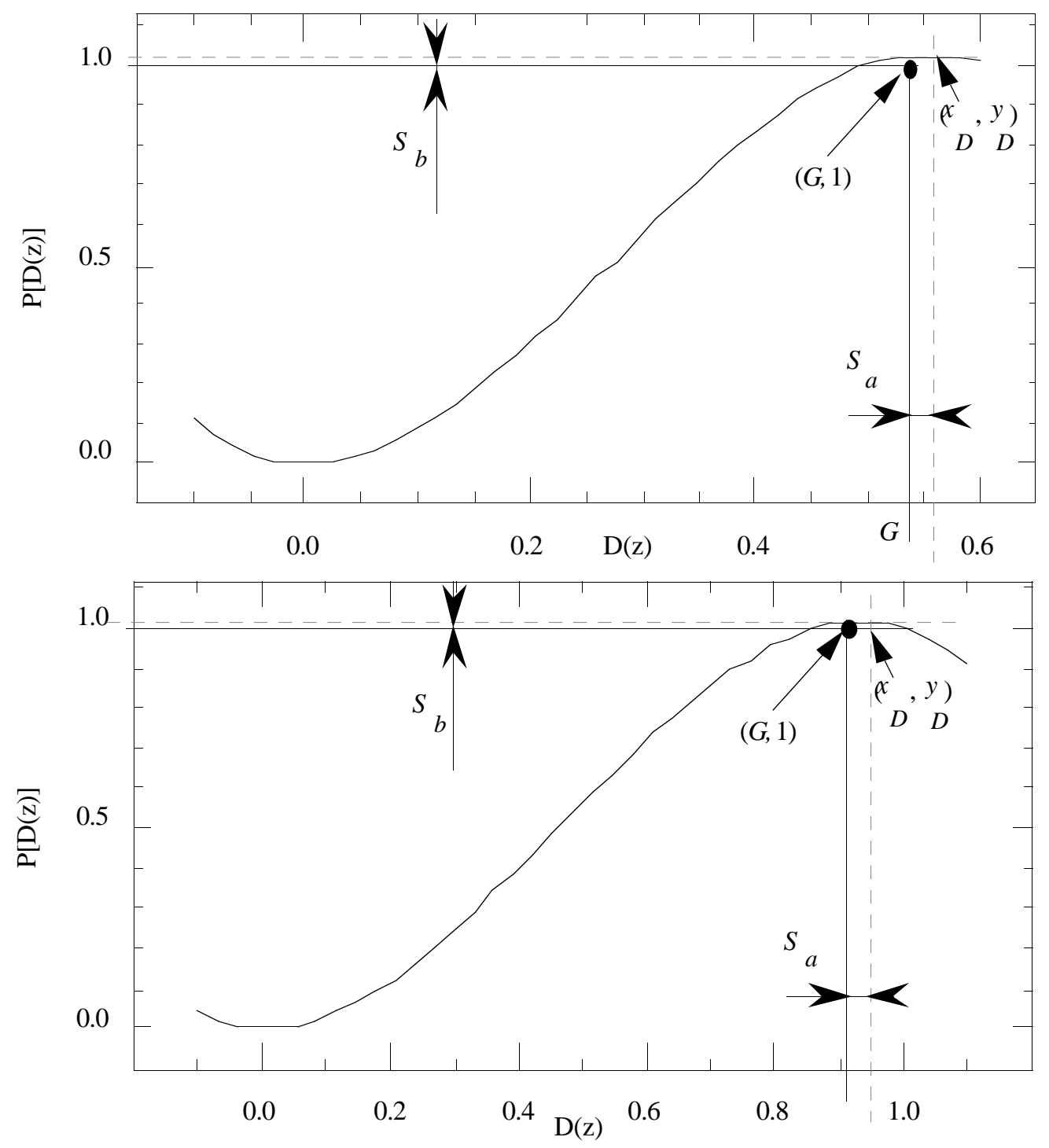

Fig.1. Sharpening polynomials $y=x\left(-12 x^{2}+10 x\right)$ and $y=x\left(-2.5 x^{2}+3.5 x\right)$ computed by (8).

TABLE 1.

RESULTS OF 3 LOW-PASS FILTER DESIGN [5]. NORMALIZED CUT-OFF FREQUENCIES ARE 0.15625 AND 0.1875.

OTHER PARAMETERS: $L=2, B=9, K=-3, F=512$.

\begin{tabular}{lllllll}
\hline $\begin{array}{l}\text { Overall } \\
\text { Filter } \\
\text { Length }\end{array}$ & $\begin{array}{l}\text { Infinite precision } \\
\text { solution [3] }\end{array}$ & $\begin{array}{l}\text { Optimized } \\
\text { with GA [11] }\end{array}$ & $\begin{array}{l}\text { Sub-filter } \\
\text { Length }\end{array}$ & $\begin{array}{l}\text { Infinite precision } \\
\text { sharpening }\end{array}$ & $\begin{array}{l}\text { Proposed } \\
\text { P2-filter } \\
\text { sharpening }\end{array}$ & $\begin{array}{l}\text { P2-filter } \\
\text { sharpening } \\
\text { polynomial }\end{array}$ \\
\hline & & & & & & \\
57 & -36.90 & -34.40 & 19 & -21.65 & -25.23 & $\mathrm{y}=\mathrm{x}\left(-12 \mathrm{x}^{2}+10 \mathrm{x}\right)$ \\
99 & -56.41 & -38.24 & 33 & -40.25 & -40.50 & $\mathrm{y}=\mathrm{x}\left(-12 \mathrm{x}^{2}+10 \mathrm{x}\right)$ \\
123 & -67.37 & -36.46 & 41 & -46.63 & -47.70 & $\mathrm{y}=\mathrm{x}\left(-2.5 \mathrm{x}^{2}+3.5 \mathrm{x}\right)$
\end{tabular}

